\title{
CENTRALIZERS OF BCI-ALGEBRAS
}

\author{
A. NAJAFI, A. BORUMAND SAEID, AND E. ESLAMI
}

Received 18 May, 2018

\begin{abstract}
In this paper, the concept of the centralizer of a subset (an element) of a BCI-algebra by using commutators is given. The connection between commutative ideals with commutators are considered. Also the pseudo center of a BCI-algebra is defined and the relationships between center and pseudo center in BCI-algebras are discussed. Following the concept of the centralizer, we introduce C-closed subalgebras of a BCI-algebra and discuss some characteristics of these subalgebras. Finally, we define central ideal and derived ideal of a BCI-algebra and the relationship between central ideal, derived ideal and other ideals of BCI-algebras are investigated.
\end{abstract}

2010 Mathematics Subject Classification: 03G25; 06F35

Keywords: BCI-algebras, centralizer, (pseudo) center, C-closed subalgebras, central (derived) ideal

\section{INTRODUCTION}

K. Iséki introduced the concept of BCI-algebra in 1966 [13]. From then some mathematicians studied and developed many concepts in this algebraic structure, for instance, T. Lei and C. Xi [20] showed that each p-semisimple BCI-algebra can be converted to an Abelian group and conversely each Abelian group is converted to a BCI-algebra. S.A. Bhatti and M.A. Chaudhry introduced the concept of the center of a BCI algebra based on the center of a group [1] and showed that the center of a p-semisimple BCI-algebra is itself. Unlike Abelian group, this is not true in the case of BCI-algebras. For example the center of a BCK-algebra $X$ is $\{0\}$. This motivates us to define pseudo center of a BCI-algebra which not only covers the mentioned deficiencies but offers a number of advantages with respect to the center. The map $\phi(x)=0 * x$, was formally introduced in [8] for $\mathrm{BCH}$-algebras, but earlier it was used in [4] and [3] to investigate some classes of BCI-algebras connected with groups. In $\mathrm{BCI}$-algebras which are quasigroups, that is, BCI-algebras isotopic to commutative groups [1], any finite subset of such BCI-algebra is an ideal if and only if it is a subgroup of the corresponding group. Any group $G$ in which the square of every element is the identity (i.e. a Boolean group) is a BCI-algebra. In [10] is proved that a BCI-algebra $(X, *, 0)$ is a Boolean group if it has a neutral element (i.e. if 
$0 * x=x * 0=x$ for all $x \in X)$ or if it is associative. Also every para-associative BCI-algebra is a Boolean group [2,6].

Since centralizer and center are two important notions, we extend these two notions to these BCI-algebras and discuss further properties of these concepts. We use the notions of pseudo center and centralizer in BCI-algebras to develop other new concepts such as idealizer and normalizer in these structures. One of the main motivations for defining pseudo center in BCI-algebras is proving similar Lagrange and Sylow theorems if possible. The C-dimension theory, a new and interesting concept has been of interest to many mathematicians recently. The theory has been developed using centralizers in some algebraic structures including groups and rings. The concept of C-dimension in these structures is defined as the length of the longest nested chain of the centralizers, which is closely related to the general theory of groups and rings. This means that if two groups have the same general theory, especially if they are elementary equivalent, they have the same C-dimension. The converse is also correct under certain conditions. Investigation of the concept of Cdimension in BCI-algebras using centralization could be an interesting subject for further studies.

In this paper, we present a definition for the centralizer of an arbitrary element in BCI-algebras on based commutators. We define also the notion of the centralizer of a subset of a BCI-algebra, give several characterizations of it and prove that the class of C-closed subalgebras of a BCI-algebra $X$ is a commutative monoid and a lower semi-lattice. We illustrate also these notions by some examples. Finally, we present the concepts of central ideal and derived ideal of a BCI-algebra and some properties of these notions are investigated. We verify some useful properties of these ideals in BCI-algebras such as relation between central ideal and derived ideal with radical of $X$.

\section{PRELIMINARIES}

By a BCI-algebra we mean an algebra $(X, *, 0)$ of type $(2,0)$ satisfying the following axioms: for all $x, y, z \in X$,

(BCI1) $((x * y) *(x * z)) *(z * y)=0$,

(BCI2) $(x *(x * y)) * y=0$,

(BCI3) $x * x=0$,

(BCI4) $x * y=y * x=0$ implies $x=y$.

A partial ordering $\leq$ on $X$ can be defined by $x \leq y$ if and only if $x * y=0$. In any BCI-algebra $X$ for all $x, y \in X$, the following hold:

(1) $(x * y) * z=(x * z) * y$,

(2) $x *(x *(x * y))=x * y$,

(3) $x * 0=x$,

(4) $x \leq y$ imply that $x * z \leq y * z$ and $z * y \leq z * x$,

(5) $(x * z) *(y * z) \leq x * y$. 
A BCI-algebra $X$ is said to be p-semisimple if $0 *(0 * x)=x$, for all $x \in X$. A nonempty subset $S$ of a BCI-algebra $X$ is called a subalgebra of $X$, if $x * y \in S$ whenever $x, y \in S$. A nonempty subset $I$ of a BCI-algebra $X$ is called an ideal if: (i) $0 \in I$ (ii) $x * y \in I$ and $y \in I$ imply $x \in I$ for all $x, y \in X$. An ideal $I$ of a BCI-algebra $X$ is called closed if $0 * x \in X$, for all $x \in X$. An element $x$ in a BCI-algebra $X$ is called a positive element if it satisfies $0 * x=0$. A BCI-algebra $X$ is called commutative if $x \leq y$ implies $x \wedge y=x$, where $x \wedge y=y *(y * x)$, for all $x, y \in X$. A BCI-algebra $X$ is called associative if $x *(y * z)=(x * y) * z$, for all $x, y, z \in X$. Let $I$ be an ideal of a BCI-algebra $X$, then the relation $\theta$ defined by $(x, y) \in \theta$ if and only if $x * y \in I$ and $y * x \in I$ is a congruence relation on $X$. Let $x / I$ denote the class of $x \in X$, then $0 / I=I$. Assume that $X / I=\{x / I: x \in X\}$. Then $(X / I, *, 0 / I)$ is a BCI-algebra, where $x / I * y / I=(x * y) / I$, for all $x, y \in X$ (see [11,14]).

In what follows, $(X, *, 0)$ or simply $X$ would mean a BCI-algebra, unless otherwise specified.

Definition 1. i) ([20]). The set $\{x \in X: 0 *(0 * x)=x\}=\left\{x \in X: \phi^{2}(x)=x\right\}$ is called the center of $X$ and is denoted by $C(X)$.

ii) ([1]). An element $x_{0} \in X$ is said to be an initial element of $X$, if $x \leq x_{0}$ implies $x=x_{0}$. Let $I_{x}$ denote the set of all initial elements of $X$. We call it the center of $X$.

iii) ([15-18]). Let $x, y$ be two elements of $X$. Then the element $((y \wedge x) *(x \wedge y)) *$ $(0 *(x * y))$ of $X$ is called a pseudo-commutator of $x$ and $y$ and is denoted by $[x, y]$.

iv) ([17]). For nonempty subsets $A$ and $B$ of $X$ the commutator of $A$ and $B$ is the set of all finite $*$-products of commutators of kind $[a, b]$ with $a \in A$ and $b \in B$.

$$
[A, B]=\left\{\left[a_{i 1}, b_{j_{1}}\right] *\left[a_{i 2}, b_{j_{2}}\right] * \ldots *\left[a_{i_{n}}, b_{j_{n}}\right]: a_{i k} \in A, b_{j_{l}} \in B, n \in N\right\} .
$$

When $A=B=X,[X, X]$ is called the commutator subalgebra or the derived subalgebra of $X$ and denoted by $X^{\prime}$. Therefore

$X^{\prime}=\left\{x_{1} * x_{2} * \ldots * x_{n}: n \geq 1\right.$, each $x_{i}$ is a pseudo-commutator in $\left.X\right\}$.

v) ([11]). An element $x$ of $X$ is a nilpotent element if $0 * x^{n}=0$ for some positive integer $n$, where $x * y^{n}=\underbrace{(\ldots((x * y) * y) * \ldots) * y}_{n \text {-times }}$. If every $x$ in $X$ is nilpotent, then $X$ is called a nilpotent BCI-algebra. For every positive integer $k$, we define $N_{k}(X)=\left\{x \in X: 0 * x^{k}=0\right\}$. The intersection of all maximal ideals of a BCI-algebra $X$ is called the radical of $X$ and is denoted by $\operatorname{Rad}(X)$.

Theorem 1 ([11]). A closed ideal I of $X$ is a commutative ideal if and only if the quotient algebra X/I is a commutative BCI-algebra.

Theorem 2 ([15,17]). $X$ is commutative if and only if $X^{\prime}=\{0\}$. 
Theorem 3 ([11]). Let $S$ be a nonempty subset of $X$ and let

$$
A=\left\{x \in X:\left(\ldots\left(\left(x * a_{1}\right) * a_{2}\right) * \ldots\right) * a_{n}=0, \text { for some } a_{1}, a_{2}, \ldots, a_{n} \in S\right\} .
$$

Then $\langle S\rangle=A \cup\{0\}$. Especially, if $S$ contains a positive element of $X$, or if $S$ contains a nilpotent element of $X$, then $<S>=A$.

Lemma 1. i) ([17]). Let $f$ be a homomorphism from $X$ to a BCI-algebra $Y$. Then $f([x, y])=[f(x), f(y)]$, for all $x, y \in X$.

ii) ([18]). $[x, y]$ is a positive element of $X$, for all $x, y \in X$.

iii) ([11]). $0 *(x * y)=(0 * x) *(0 * y)$, for all $x, y \in X$, i.e., $\phi$ is a homomorphism.

Theorem 4 ([11]). The following two types of abstract systems are equivalent: p-semisimple BCI-algebra and Abelian group.

In the following lemma we show that the center of derived subalgebra $C\left(X^{\prime}\right)$ is always $\{0\}$.

Lemma 2. $\quad$ i) $x \in C(X)$ if and only if $a *(a * x)=x$, for all $a \in X$.

ii) If $x \in C(X)$, then $\phi(y * x)=x * y$, for all $y \in X$.

iii) $C\left(X^{\prime}\right)=\{0\}$.

Proof. i) Let $x \in C(X)$. Then $0 *(0 * x)=x$. Since $(a *(a * x)) * x=0, a *(a * x) \leq x$. Conversely, $(a *(a * x)) * x=0$, then $0 *((a *(a * x)) * x)=0 * 0=0$. Hence $(0 *(a *(a * x))) *(0 * x)=0$. Therefore $(0 *(0 * x)) *(a *(a * x))=0$. Whence $x *(a *(a * x))=0$. Hence $x \leq(a *(a * x))$. Then $a *(a * x)=x$, for all $a \in X$. If $a *(a * x)=x$, for any $a \in X$, then $0 *(0 * x)=x$. Therefore $x \in C(X)$.

ii) Suppose that $x \in C(X)$. Then

$$
\phi(y * x)=0 *(y * x)=(0 * y) *(0 * x)=(0 *(0 * x)) * y=x * y .
$$

iii) Let $x \in C\left(X^{\prime}\right)$. Then $x \in X^{\prime}$ and $0 *(0 * x)=x$. Thus there exist $a_{i}, b_{i} \in X$ such that $x=\prod\left[a_{i}, b_{i}\right]$. Hence

$$
\begin{aligned}
x & =0 *(0 * x)=0 *\left(0 * \prod\left[a_{i}, b_{i}\right]\right) \\
& =\left(0 *\left(0 *\left[a_{1}, b_{1}\right]\right)\right) *\left(0 *\left(0 *\left[a_{2}, b_{2}\right]\right)\right) * \ldots *\left(0 *\left(0 *\left[a_{n}, b_{n}\right]\right)\right)=0 * \ldots * 0=0 .
\end{aligned}
$$

Therefore, $C\left(X^{\prime}\right)=\{0\}$.

Theorem 5. Let $x, y \in X$. Then

i) $\phi([x, y])=0$,

ii) $[\phi(x), \phi(y)]=0$.

Proof. i) We first show that $[x, y] * y \leq \phi(y)$.

$$
\begin{aligned}
{[x, y] * y } & =(((x *(x * y)) *(y *(y * x))) *(0 *(x * y))) * y \\
& \leq((y *(y *(y * x))) *(0 *(x * y))) * y \\
& =((y * x) *(0 *(x * y))) * y=((y * x) * y) *(0 *(x * y))
\end{aligned}
$$




$$
\begin{aligned}
& =((y * y) * x) *(0 *(x * y))=(0 * x) *((0 * x) *(0 * y)) \\
& \leq 0 * y=\phi(y) .
\end{aligned}
$$

Therefore $0=([x, y] * y) *(0 * y) \leq[x, y] * 0=[x, y]$. So $\phi([x, y])=0 *[x, y]=0$. ii) By substitute $\phi$ for $f$ in Lemma 1 we obtain $0=\phi([x, y])=[\phi(x), \phi(y)]$.

W. A. Dudek presents a new method for studying the ideals and centralizer of 0 element based on the map $\phi$ in $\mathrm{BCI} / \mathrm{BCH} / \mathrm{BCC}$-algebra $(X, *, 0)$ and some useful facts on these notion are proved in [2-9]. He defines the centralizer of 0 element in $X$ by $Z_{0}=\{x \in X: x * 0=0 * x\}=\{x \in X: \phi(x)=x\}$. It is shown that if $(X, *, 0)$ is a $p$ semisimple BCI-algebra, then $(X, ., 0)$ is an Abelian group, where $x \cdot y=x *(0 * y)$ for $x, y \in X$ and conversely, if $(X, ., 0)$ is an Abelian group, then $(X, *, 0)$ with $x * y=x \cdot y^{-1}$ is a $p$-semisimple BCI-algebra [3]. So we expect that the centralizer of 0 to be a fixed element such as neutral element in Abelian groups. But in the following example we see that this is not true in general.

Example 1. We consider Abelian group $\left(Z_{3},+, 0\right)$ and adjoint $p$-semisimple BCIalgebra $\left(Z_{3}^{a d}, *, 0\right)$ of it with the following Cayley table:

\begin{tabular}{c|ccc}
+ & 0 & $a$ & $b$ \\
\hline 0 & 0 & $a$ & $b$ \\
$a$ & $a$ & $b$ & 0 \\
$b$ & $b$ & 0 & $a$
\end{tabular}

\begin{tabular}{c|ccc}
$*$ & 0 & $a$ & $b$ \\
\hline 0 & 0 & $b$ & $a$ \\
$a$ & $a$ & 0 & $b$ \\
$b$ & $b$ & $a$ & 0
\end{tabular}

By definition of centralizer of an element in Abelian group $\left(Z_{3},+, 0\right)$ as neutral element 0 we have $Z_{0}=\left\{x \in Z_{3}: x+0=0+x\right\}=Z_{3}$ and in adjoint $p$-semisimple BCI-algebra $\left(Z_{3}^{a d}, *, 0\right)$ we obtain $Z_{0}=\left\{x \in Z_{3}: x * 0=0 * x\right\}=\{0\}$.

We are trying to resolve this disagreement. By attention to $\phi(x)$, we would like to consider centralizer from another perspective.

\section{Centralizer of a Subset in BCI-Algebras}

In this section, we introduce the notion of centralizer of a subset of BCI-algebras by using commutators and study it in detail.

Definition 2. Suppose that $S$ is a nonempty subset of $X$. The centralizer of $S$ in $X$ is defined to be $\{x \in X:[x, s]=[s, x]=0, \forall s \in S\}$ and denoted by $C_{X}(S)$.

When $S=\{x\}$ is a singleton set, then $C_{X}(\{x\})$ can be abbreviated to $C_{X}(x)$. Symbolically,

$$
C_{X}(x)=\{y \in X:[x, y]=[y, x]=0\} .
$$

$C_{X}(x)$ is a nonempty set, because $[x, 0]=[0, x]=[x, x]=0$, for any $x \in X$. Specifically, $0, x \in C_{X}(x)$. 
Example 2. Let $X=\{0, a, b, c, d\}$ be a $B C I$-algebra with the following Cayley table:

\begin{tabular}{l|lllll}
$*$ & 0 & $a$ & $b$ & $c$ & $d$ \\
\hline 0 & 0 & 0 & 0 & $c$ & $c$ \\
$a$ & $a$ & 0 & 0 & $c$ & $c$ \\
$b$ & $b$ & $b$ & 0 & $d$ & $c$ \\
$c$ & $c$ & $c$ & $c$ & 0 & 0 \\
$d$ & $d$ & $d$ & $c$ & $b$ & 0
\end{tabular}

By simple calculations we obtain $C_{X}(0)=C_{X}(c)=C_{X}(d)=X, C_{X}(a)=\{0, a, c, d\}$ and $C_{X}(b)=\{0, b, c, d\}$. For $S=\{0, a, b\}$ we obtain $C_{X}(S)=\{0, c, d\}$.

Now we describe the relation between centralizer of a set with centralizer of constituent elements.

Theorem 6. For subset $S$ of $X, C_{X}(S)=\bigcap_{a \in S} C_{X}(a)$.

Proof.

$$
\begin{aligned}
x \in C_{X}(S) & \Leftrightarrow \forall a \in S,[x, a]=[a, x]=0 \\
& \Leftrightarrow \forall a \in S, x \in C_{X}(a) \\
& \Leftrightarrow x \in \bigcap_{a \in S} C_{X}(a) .
\end{aligned}
$$

Lemma 3. Suppose that $I$ is an ideal of $X$ and $a, b \in X$. Then

i) $C_{X}(0)=X$,

ii) $a \in C_{X}(b)$ iff $b \in C_{X}(a)$,

iii) $C_{X}(a) / I \subseteq C_{X / I}(a / I)$.

Proof. i) $C_{X}(0)=\{x \in X:[x, 0]=[0, x]=0\}=X$.

ii) $a \in C_{X}(b)$ if and only if $[a, b]=[b, a]=0$ if and only if $b \in C_{X}(a)$.

iii) Let $b / I \in C_{X}(a) / I$. Then $b \in C_{X}(a)$ and hence $[a, b]=[b, a]=0$. Therefore $[a, b] / I=[b, a] / I=0 / I$. But $[a, b] / I=[a / I, b / I]$ and $[b, a] / I=[b / I, a / I]$. Then $[a / I, b / I]=[b / I, a / I]=0 / I$. So $b / I \in C_{X / I}(a / I)$. Hence $C_{X}(a) / I \subseteq C_{X / I}(a / I)$.

Lemma 4. Let $f \in \operatorname{Aut}(X)$. Then $f\left(C_{X}(a)\right)=C_{X}(f(a))$, for every $a \in X$.

Proof. Let $y \in f\left(C_{X}(a)\right)$. Then there exists $x \in C_{X}(a)$ such that $y=f(x)$. Since $x \in C_{X}(a),[x, a]=[a, x]=0$. Therefore, $f([x, a])=f([a, x])=f(0)=0$ and hence $[f(x), f(a)]=[f(a), f(x)]=0$. Thus $y=f(x) \in C_{X}(f(a))$. i.e., $f\left(C_{X}(a)\right) \subseteq C_{X}(f(a))$. If $y=f(x) \in C_{X}(f(a))$, then $[f(x), f(a)]=[f(a), f(x)]=0$. Therefore $f([x, a])=$ $f([a, x])=f(0)=0$. But $f$ is one to one, then $[x, a]=[a, x]=0$. Hence $x \in C_{X}(a)$ 
and so $y=f(x) \in f\left(C_{X}(a)\right)$, that is, $C_{X}(f(a)) \subseteq f\left(C_{X}(a)\right)$. Thus, $f\left(C_{X}(a)\right)=$ $C_{X}(f(a))$.

Corollary 1. For subset $S$ of $X$ and $f \in A u t(X), f\left(C_{X}(S)\right)=C_{X}(f(S))$.

Proof.

$$
\begin{aligned}
f\left(C_{X}(S)\right) & =f\left(\bigcap_{a \in S} C_{X}(a)\right) \\
& =\bigcap_{a \in S} f\left(C_{X}(a)\right) \\
& =\bigcap_{a \in S} C_{X}(f(a)) \\
& =C_{X}(f(S)) .
\end{aligned}
$$

In the following two theorems, some of the properties of operator $C_{X}$, such as symmetry and decreasing are examined.

Theorem 7. Suppose that $S, T$ are two subsets of $X$. Then

i) $S \subseteq C_{X}(T)$ iff $T \subseteq C_{X}(S)$,

ii) If $S \subseteq T$, then $C_{X}(T) \subseteq C_{X}(S)$.

Proof. i) Let $S \subseteq C_{X}(T)$ and let $t \in T$. To show that $t \in C_{X}(S)$ we must show that for all $s \in S,[t, s]=[s, t]=0$. Suppose that $s$ is an arbitrary element of $S$. Therefore $s \in C_{X}(T)$. By definition $[t, s]=[s, t]=0$, for every $t \in T$. Hence $t \in C_{X}(S)$. By symmetry if $T \subseteq C_{X}(S)$, then we see $S \subseteq C_{X}(T)$.

ii) Let $S \subseteq T$. If $x \in C_{X}(T)$, then $[x, t]=[t, x]=0$, for all $t \in T$. Since $S \subseteq T$, for all $s \in S,[x, s]=[s, x]=0$. Hence $x \in C_{X}(S)$.

Theorem 8. Let $S$ be a subset of $X$. Then

i) $S \subseteq C_{X}\left(C_{X}(S)\right)$,

ii) $C_{X}\left(C_{X}\left(C_{X}(S)\right)\right)=C_{X}(S)$.

Proof. i) Let $x \in S$. Then $x \in C_{X}\left(C_{X}(S)\right)$ iff for all $s \in C_{X}(S)$ we have $[x, s]=$ $[s, x]=0$. Let $s$ be an arbitrary element of $C_{X}(S)$. Then $\left[s, s^{\prime}\right]=\left[s^{\prime}, s\right]=0$, for all $s^{\prime} \in S$. Since $x \in S,[x, s]=[s, x]=0$. Therefore $S \subseteq C_{X}\left(C_{X}(S)\right)$.

ii) Since $S \subseteq C_{X}\left(C_{X}(S)\right)$, it follows that $C_{X}\left(C_{X}\left(C_{X}(S)\right)\right) \subseteq C_{X}(S)$. Also we obtain $C_{X}(S) \subseteq C_{X}\left(C_{X}\left(C_{X}(S)\right)\right)$ by putting $C_{X}(S)$ instead of $S$ in (i). Hence $C_{X}(S)=$ $C_{X}\left(C_{X}\left(C_{X}(S)\right)\right)$.

Remark 1. $C_{X}\left(C_{X}(S)\right)$ contains $S$ but is not necessarily equal, also $C_{X}(S)$ need not contain $S$. For the set $S=\{0, a, b\}$ from Example 2, we obtain $C_{X}(S)=\{0, c, d\}$ that is not contain $S$. Also $C_{X}\left(C_{X}(S)\right)=X \neq S$.

Definition 3. A subalgebra $S$ of $X$ is said to be C-closed if $S=C_{X}\left(C_{X}(S)\right)$. 
We denote by $\mathbb{C}(X)$ the set of all C-closed subalgebras of $X$. Since $C_{X}\left(C_{X}(X)\right)$ contains $X, C_{X}\left(C_{X}(X)\right)=X$. Then $\mathbb{C}(X)$ is nonempty.

Obvious that if $C_{X}(S)$ is a subalgebra of $X$, then $C_{X}(S)$ is C-closed, because $C_{X}(S)=C_{X}\left(C_{X}\left(C_{X}(S)\right)\right)$.

Example 3. Let $X=\{0, a, b, c, d\}$ be a $B C I$-algebra with the following Cayley table:

\begin{tabular}{l|lllll}
$*$ & 0 & $a$ & $b$ & $c$ & $d$ \\
\hline 0 & 0 & 0 & 0 & 0 & 0 \\
$a$ & $a$ & 0 & 0 & $a$ & 0 \\
$b$ & $b$ & $a$ & 0 & $b$ & $a$ \\
$c$ & $c$ & $c$ & $c$ & 0 & $c$ \\
$d$ & $d$ & $d$ & $d$ & $d$ & 0
\end{tabular}

$X$ has 14 subalgebra, but only 4 until of their are C-closed. The sets $S_{1}=\{0, c\}$, $S_{2}=\{0, c, d\}, S_{3}=\{0, a, b, c\}, S_{4}=X$ are subalgebras of $X$ such that

$C_{X}\left(C_{X}\left(S_{1}\right)\right)=S_{1}, C_{X}\left(C_{X}\left(S_{2}\right)\right)=S_{2}, C_{X}\left(C_{X}\left(S_{3}\right)\right)=S_{3}$ and $C_{X}\left(C_{X}\left(S_{4}\right)\right)=S_{4}$.

Hence $\mathbb{C}(X)=\{\{0, c\},\{0, c, d\},\{0, a, b, c\}, X\}$.

Now, we move to the study of C-closed subalgebras of BCI-algebras and it consequences.

Theorem 9. If $S, T$ are $C$-closed subalgebras of $X$, then $S \cap T$ is a $C$-closed subalgebra of $X$.

Proof. Let $S, T$ be C-closed subalgebras of $X$. Clearly, $S \cap T$ is a subalgebra of $X$. Since $C_{X}\left(C_{X}(S \cap T)\right)$ contains $S \cap T$, it is sufficient to show that $C_{X}\left(C_{X}(S \cap T)\right) \subseteq(S \cap$ $T)$. But $S \cap T \subseteq S$ and $S \cap T \subseteq T$, then $C_{X}(S) \subseteq C_{X}(S \cap T)$ and $C_{X}(T) \subseteq C_{X}(S \cap T)$. Hence $C_{X}\left(C_{X}(S \cap T)\right) \subseteq C_{X}\left(C_{X}(S)\right)=S$ and $C_{X}\left(C_{X}(S \cap T)\right) \subseteq C_{X}\left(C_{X}(T)\right)=T$. That means $C_{X}\left(C_{X}(S \cap T)\right) \subseteq(S \cap T)$.

This proves that the intersection of any two C-closed subalgebra of $X$ is again an C-closed subalgebra of $X$. The above theorem can be generalized to intersection of any family of C-closed subalgebra of $X$.

Remark 2. A C-closed subalgebra of a C-closed subalgebra is again C-closed.

Corollary 2. If $X$ is commutative, then $X$ is only subalgebra that is $C$-closed.

Proof. Let $X$ be commutative. Then for every proper subalgebra $S$ of $X, C_{X}(S)=$ $X$. Therefore $C_{X}\left(C_{X}(S)\right)=C_{X}(X)=X \neq S$, for every proper subalgebra $S$ of $X$. If $S=X$, then $C_{X}\left(C_{X}(X)\right)=C_{X}(X)=X$.

The following example shows that the converse of Corollary 2 is not correct in general. 
Example 4. Let $X=\{0, a, b, c, d\}$ be a $B C I$-algebra in which $*$ operation is defined by the following table:

\begin{tabular}{l|lllll}
$*$ & 0 & $a$ & $b$ & $c$ & $d$ \\
\hline 0 & 0 & 0 & 0 & 0 & 0 \\
$a$ & $a$ & 0 & 0 & 0 & 0 \\
$b$ & $b$ & $a$ & 0 & 0 & 0 \\
$c$ & $c$ & $a$ & $a$ & 0 & 0 \\
$d$ & $d$ & $c$ & $b$ & $a$ & 0
\end{tabular}

By routine calculations we obtain $\mathbb{C}(X)=\{X\}$. But $X$ is not commutative, because $b \wedge c=a \neq c \wedge b=b$.

$(\mathbb{C}(X), \cap, X)$ is a commutative monoid. $\mathbb{C}(X)$ is closed under $\cap$ and for any $S \in \mathbb{C}(X), S \cap X=S$. Moreover the operation $\cap$ is commutative and associative. Also $(\mathbb{C}(X), \cap)$ forms a lower semi-lattice with respect to $\subseteq$. Indeed, $(\mathbb{C}(X), \subseteq)$ is a partially ordered set and for any $S, T \in \mathbb{C}(X)$ we have inf $\{S, T\}=S \cap T$.

In the following lemma we examine the conditions under which the converse of the Theorem 7 (ii) is also true.

Lemma 5. If $S \in \mathbb{C}(X)$ and $C_{X}(S) \subseteq C_{X}(T)$, then $T \subseteq S$.

Proof. Suppose that $C_{X}(S) \subseteq C_{X}(T)$. Then $C_{X}\left(C_{X}(T)\right) \subseteq C_{X}\left(C_{X}(S)\right)$. Since $S \in \mathbb{C}(X), C_{X}\left(C_{X}(S)\right)=S$, hence $T \subseteq C_{X}\left(C_{X}(T)\right) \subseteq S$.

\section{THE PSEUDO CENTER OF BCI-ALGEBRAS}

In this section, at first, we recall that the center of a BCI-algebra is defined in several different ways such as Definition 1, but with common results. However, the logical reasons for this definitions is not clear, it is famed that in algebraic structures including groups, rings and Lie algebras the notion of center is defined based on commutators $[10,19]$. These motivate us to introduce a new notion of center in BCI-algebras without using the commutators. This concept is different from the center of BCI-algebras previously defined but it is consistent with the center of other mentioned algebras. The new proposed center definition is more general and reliable and is called pseudo center in this manuscript.

Definition 4. The set $\{x \in X:[x, y]=[y, x]=0, \forall y \in X\}$ is called the pseudo center of $X$ and is denoted by $Z(X)$.

Obviously, $0 \in Z(X)$.

Example 5. i) For Example 2, $Z(X)=\{0, c, d\}, C(X)=\{0, c\}$ and $X^{\prime}=\{0, a\}$. ii) Let $X=\{0, a, b, c, d\}$ be a $B C I$-algebra with the following Cayley table: 


\begin{tabular}{l|lllll}
$*$ & 0 & $a$ & $b$ & $c$ & $d$ \\
\hline 0 & 0 & 0 & 0 & 0 & 0 \\
$a$ & $a$ & 0 & $a$ & 0 & $a$ \\
$b$ & $b$ & $b$ & 0 & $b$ & 0 \\
$c$ & $c$ & $a$ & $c$ & 0 & $c$ \\
$d$ & $d$ & $d$ & $d$ & $d$ & 0
\end{tabular}

By simple calculations we obtain $Z(X)=\{0, a, c\}, C(X)=\{0\}$ and $X^{\prime}=\{0, b\}$. Indeed, $Z(X)$ is commutative part of $X$, and $X^{\prime}$ evaluates non commutative part and commutative part of $X$ from each other.

Theorem 10. $X$ is commutative if and only if $Z(X)=X$.

Proof. Let $X$ be commutative and $x \in X$. Then $[x, y]=[y, x]=0$, for every $y \in X$. So $x \in Z(X)$ and hence $X \subseteq Z(X)$. Obviously $Z(X) \subseteq X$. Thus $Z(X)=X$.

Conversely, let $Z(X)=X$. Then $[x, y]=[y, x]=0$, for all $x, y \in X$. Therefore $X$ is a commutative BCI-algebra.

Corollary 3. The following conditions are equivalent:

i) $X$ is commutative,

ii) $Z(X)=X$,

iii) $X^{\prime}=\{0\}$.

Proof. i) $\leftrightarrow$ ii) follows directly from Theorem 10 .

ii) $\leftrightarrow$ iii) Let $Z(X)=X$. Then $X$ is commutative. By Theorem $6, X^{\prime}=\{0\}$.

Conversely, let $X^{\prime}=\{0\}$. Therefore, $X$ is commutative. Hence $Z(X)=X$.

Remark 3. $Z(X)$ is neither an ideal nor a subalgebra of $X$, in general. From Example 4 by routine calculations we obtain that $Z(X)=\{0, a, d\}$ is not a subalgebra of $X$ because $a, d \in Z(X)$ but $d * a=c \notin Z(X)$ also $Z(X)$ is not an ideal of $X$ because $c * a \in Z(X)$ and $a \in Z(X)$, but $c \notin Z(X)$. Also, $C_{X}(I)$ for ideal $I$ is not an ideal (a subalgebra) of $X$, in general. For instance, in Example $4, X$ is an ideal of $X$, but $C_{X}(X)=Z(X)$ is not an ideal (a subalgebra) of $X$.

In the following proposition we describe the relationship between center and pseudo center in BCI-algebras.

Proposition 1. $C(X) \subseteq Z(X)$.

Proof. Suppose that $x \in C(X)$. Then $0 *(0 * x)=x$. We must show that for all $y \in X,[x, y]=[y, x]=0$. But

$$
\begin{aligned}
{[x, y] c } & =((x *(x * y)) *(y *(y * x))) *(0 *(x * y))=((x *(x * y)) *(x)) *(0 *(x * y)) \\
& =((x * x) *(x * y)) *(0 *(x * y))=(0 *(x * y)) *(0 *(x * y))=0 .
\end{aligned}
$$


Also

$$
\begin{aligned}
{[y, x] } & =((y *(y * x)) *(x *(x * y))) *(0 *(y * x))=(x *(x *(x * y))) *(0 *(y * x)) \\
& =(x * y) *(x * y)=0 .
\end{aligned}
$$

Therefore $x \in Z(X)$. Hence $C(X) \subseteq Z(X)$.

Remark 4. In Example 4 we see $Z(X)=\{0, a, d\}$ and $C(X)=\{0\}$. Then the equality of Proposition 1 does not hold, in general.

As immediate consequences of Definition 2 and Definition 4 we obtain:

Theorem 11. Suppose that $a, b \in X$ and $X_{1}, X_{2}$ are subsets of $X$. Then

i) $a \in Z(X)$ if and only if $C_{X}(a)=X$,

ii) $Z(X)=\bigcap_{a \in X} C_{X}(a)$,

iii) If $X_{1} \subseteq X_{2}$, then $Z\left(X_{2}\right) \subseteq Z\left(X_{1}\right)$,

iv) $C_{X}(a) \subseteq C_{X}(b)$ if and only if $b \in Z\left(C_{X}(a)\right)$,

v) $Z(X) \subseteq C_{X}(a)$, for every $a \in X$,

vi) $X$ is commutative if and only if $C_{X}(a)=X$, for every $a \in X$,

vii) $C_{X}(X)=Z(X)$,

viii) $C_{X}(a)=C_{X}(b)$ if and only if $Z\left(C_{X}(a)\right)=Z\left(C_{X}(b)\right)$.

Proof. i) Let $a \in Z(X)$. Then for all $x \in X,[x, a]=[a, x]=0$. Obviously, $C_{X}(a) \subseteq$ $X$. Now, let $x \in X$. Thus $[x, a]=[a, x]=0$. Therefore $x \in C_{X}(a)$, that is, $X \subseteq C_{X}(a)$. Hence $C_{X}(a)=X$. Conversely, let $C_{X}(a)=X$. Since $C_{X}(a)=\{b \in X:[a, b]=[b, a]=$ $0\}=X$, it follows that $a \in Z(X)$.

ii) Let $x \in Z(X)$. Then $[x, a]=[a, x]=0$ for every $a \in X$. So $x \in C_{X}(a)$, for every $a \in X$. That means $x \in \bigcap_{a \in X} C_{X}(a)$. Therefore $Z(X) \subseteq \bigcap_{a \in X} C(a)$.

Conversely, let $x \in \bigcap_{a \in X} C_{X}(a)$. Then $x \in C_{X}(a)$ for every $a \in X$. Hence $[x, a]=$ $[a, x]=0$ for every $a \in X$. Then $x \in Z(X)$. So $\bigcap_{a \in X} C_{X}(a) \subseteq Z(X)$.

iii) Let $x \in Z\left(X_{2}\right)$. Then for every $y \in X_{2},[x, y]=[y, x]=0$. Since $X_{1} \subseteq X_{2}$, for every $y \in X_{1}$ we have $[x, y]=[y, x]=0$. Therefore $x \in Z\left(X_{1}\right)$.

iv) Let $C_{X}(a) \subseteq C_{X}(b)$. Then for any $x \in C_{X}(a), x \in C_{X}(b)$. Hence $[x, b]=[b, x]=$ 0 , for any $x \in C_{X}(a)$. Therefore $b \in Z\left(C_{X}(a)\right)$. Conversely, let $b \in Z\left(C_{X}(a)\right)$ and $x \in C_{X}(a)$. Therefore $[x, b]=[b, x]=0$. Hence $x \in C_{X}(b)$. Then $C_{X}(a) \subseteq C_{X}(b)$.

v) Since $Z(X)=\bigcap_{a \in X} C_{X}(a)$, it follows that $Z(X) \subseteq C_{X}(a)$, for any $a \in X$.

vi) $X$ is commutative iff $Z(X)=X$ iff $X=\bigcap_{a \in X} C_{X}(a)$ iff $X=C_{X}(a)$, for every $a \in X$.

vii) $C_{X}(X)=\{x \in X:[x, y]=[y, x]=0$, for all $y \in X\}=Z(X)$.

viii) Obviously, if $C_{X}(a)=C_{X}(b)$, then $Z\left(C_{X}(a)\right)=Z\left(C_{X}(b)\right)$. Conversely, let $Z\left(C_{X}(a)\right)=Z\left(C_{X}(b)\right)$. Since $a \in Z\left(C_{X}(a)\right), a \in Z\left(C_{X}(b)\right)$. Then $C_{X}(b) \subseteq C_{X}(a)$. Similarly, since $b \in Z\left(C_{X}(b)\right), b \in Z\left(C_{X}(a)\right)$, then $C_{X}(a) \subseteq C_{X}(b)$. Therefore $C_{X}(a)=$ $C_{X}(b)$.

Theorem 12. Let I be an ideal of $X$. Then $Z(X) / I \subseteq Z(X / I)$. 
Proof. Let $x / I \in Z(X) / I$. Then $x \in Z(X)$ and hence for every $y \in X,[x, y]=[y, x]=$ 0 . Therefore $[x, y] / I=[y, x] / I=0 / I$. Hence $[x / I, y / I]=[y / I, x / I]=0 / I$, for every $y / I \in X / I$. So $x / I \in Z(X / I)$. Thus $Z(X) / I \subseteq Z(X / I)$.

The following example shows that the equality of Theorem 12 may not hold.

Example 6. Let $X=\{0,1,2,3,4,5\}$ be a $B C I$-algebra with the Cayley table as follows:

\begin{tabular}{l|llllll}
$*$ & 0 & 1 & 2 & 3 & 4 & 5 \\
\hline 0 & 0 & 0 & 0 & 0 & 0 & 5 \\
1 & 1 & 0 & 1 & 0 & 1 & 5 \\
2 & 2 & 2 & 0 & 2 & 0 & 5 \\
3 & 3 & 3 & 3 & 0 & 0 & 5 \\
4 & 4 & 3 & 4 & 1 & 0 & 5 \\
5 & 5 & 5 & 5 & 5 & 5 & 0
\end{tabular}

By simple calculations we obtain $Z(X)=\{0,5\}=C(X)$. For ideal $I=\{0,2\}$ of $X$ we have $X / I=\{0 / I, 1 / I, 3 / I, 4 / I, 5 / I\}$. By routine calculus we obtain $Z(X / I)=$ $\{0 / I, 4 / I, 5 / I\}$ and $Z(X) / I=\{0 / I, 5 / I\}$. Then $Z(X) / I \subsetneq Z(X / I)$.

Lemma 6. $f(Z(X)) \subseteq Z(X)$ and $f(C(X)) \subseteq C(X)$, for every $f \in \operatorname{Aut}(X)$.

Proof. Let $y \in f(Z(X))$. Then there exists $x \in Z(X)$ such that $y=f(x)$. Since $x \in Z(X),[x, a]=[a, x]=0$, for all $a \in X$. Therefore, $f[x, a]=f[a, x]=f(0)=0$ and hence $[f(x), f(a)]=[f(a), f(x)]=0$. Since $f \in \operatorname{Aut}(X), y=f(x) \in Z(X)$. Hence $f(Z(X)) \subseteq Z(X)$.

If $y \in f(C(X))$, then exists $x \in C(X)$ such that $y=f(x)$. Since $x \in C(X)$, $0 *(0 * x)=x$. Therefore $f(x)=f(0 *(0 * x))=f(0) *(f(0) * f(x))=0 *(0 * f(x))$. Thus $y \in C(X)$. Hence $f(C(X)) \subseteq C(X)$.

Proposition 2. $\quad$ i) If $X$ is a p-semisimple, then $Z(X)=X$.

ii) If $X$ is an associative, then $Z(X)=X$.

Proof. i) Let $X$ be a p-semisimple. Since $C(X) \subseteq Z(X)$ and $C(X)=X$ we obtain $Z(X)=X$

ii) Suppose that $X$ is associative, then for any $x, y \in X$

$$
\begin{aligned}
{[x, y] } & =((x *(x * y)) *((y *(y * x)))) *(0 *(x * y)) \\
& =(((x * x) * y) *((y * y) * x)) *(x * y) \\
& =((0 * y) *(0 * x)) *(x * y) \\
& =(y * x) *(x * y)=(y * x) *(y * x)=0 .
\end{aligned}
$$

Similarly, $[y, x]=0$. Then $Z(X)=X$. 
In the following example we show that the converse of Proposition 2 is generally not correct.

Example 7. Let $X=\{0, a, b\}$ with $(*)$ be defined by the following table:

\begin{tabular}{c|ccc}
$*$ & 0 & $a$ & $b$ \\
\hline 0 & 0 & 0 & 0 \\
$a$ & $a$ & 0 & 0 \\
$b$ & $b$ & $a$ & 0
\end{tabular}

$X$ is a commutative BCI-algebra. Therefore $Z(X)=X$. But $X$ is not associative because $a=a *(a * b) \neq(a * a) * b=0$. Also $X$ is not p-semisimple because $0 *(0 * a) \neq a$.

Lemma 7. Let $X, Y$ be two BCI-algebras. Then $Z(X \times Y)=Z(X) \times Z(Y)$.

Proof. Let $(x, y) \in Z(X \times Y)$. Then for every $(a, b) \in X \times Y$, we obtain

$$
[(x, y),(a, b)]=[(a, b),(x, y)]=(0,0) .
$$

But

$$
\begin{aligned}
& {[(x, y),(a, b)]} \\
& =(((x, y) *((x, y) *(a, b))) *((a, b) *((a, b) *(x, y)))) *((0,0) *((x, y) *(a, b))) \\
& =((x *(x * a), y *(y * b)) *(a *(a * x), b *(b * y))) *(0 *(x * a), 0 *(y * b)) \\
& =((x *(x * a) *(a *(a * x))),((y *(y * b)) *(b *(b * y)))) *(0 *(x * a), 0 *(y * b)) \\
& =((x *(x * a) *(a *(a * x)) *(0 *(x * a))),((y *(y * b)) *(b *(b * y))) *(0 *(y * b))) \\
& =([x, a],[y, b]) .
\end{aligned}
$$

Since $[(x, y),(a, b)]=[(a, b),(x, y)]=(0,0),([x, a],[y, b])=([a, x],[b, y])=(0,0)$. Therefore, $[x, a]=[a, x]=0$, for any $a \in X$ and $[y, b]=[b, y]=0$, for any $b \in Y$. Hence $x \in Z(X)$ and $y \in Z(Y)$. Then $(x, y) \in Z(X) \times Z(Y)$. That means $Z(X \times Y) \subseteq$ $Z(X) \times Z(Y)$.

Conversely, let $(x, y) \in Z(X) \times Z(Y)$. Then $x \in Z(X)$ and $y \in Z(Y)$. Therefore, for every $a \in X,[x, a]=[a, x]=0$ and for every $b \in Y,[y, b]=[b, y]=0$. Thus $[(x, y),(a, b)]=([x, a],[y, b])=(0,0)$ and $[(a, b),(x, y)]=([a, x],[b, y])=(0,0)$. So $(x, y) \in Z(X \times Y)$. Hence $Z(X) \times Z(Y) \subseteq Z(X \times Y)$. Whence $Z(X \times Y)=Z(X) \times$ $Z(Y)$.

Lemma 8. Let $S$ be a subalgebra of $X$. Then

i) $S \subseteq Z(Z(S))$,

ii) $Z(Z(Z(S)))=Z(S)$. 
Proof. i) Let $s \in S$. Then for any $x \in Z(S)$ we get $[x, s]=[s, x]=0$. Hence $s \in Z(Z(S))$. Therefore $S \subseteq Z(Z(S))$.

ii) Since $S \subseteq Z(Z(S))$, it follows that $Z(Z(Z(S))) \subseteq Z(S)$. Also by putting $Z(S)$ instead of $S$ in (i) we obtain $S \subseteq Z(Z(Z(S)))$. Hence $Z(Z(Z(S)))=Z(S)$.

Theorem 13. $Z(X) \subseteq \bigcap_{S \in \mathbb{C}(X)} S$.

Proof. Since $C_{X}(S) \subseteq X$, for any $S \in \mathbb{C}(X)$, we obtain $C_{X}(X) \subseteq C_{X}\left(C_{X}(S)\right)$. Hence for any $S \in \mathbb{C}(X), C_{X}(X) \subseteq S$. Then $Z(X)=C_{X}(X) \subseteq \bigcap_{S \in \mathbb{C}(X)} S$.

Theorem 14. Suppose that $S$ is a nonempty subset of $X$. Then the following conditions are equivalent:

i) $C_{X}(S)=X$,

ii) $S \subseteq Z(X)$,

iii) $[S, X]=[X, S]=\{0\}$.

Proof. i) $\leftrightarrow$ ii) Let $C_{X}(S)=X$ and $s \in S$. Since $s \in X=C_{X}(S)$ we obtain $[x, s]=$ $[s, x]=0$, for every $x \in X$. Therefore $s \in Z(X)$. Conversely, let $S \subseteq Z(X)$. Then $C_{X}(Z(X)) \subseteq C_{X}(S)$. But $C_{X}(Z(X))=X$ and hence $X \subseteq C_{X}(S)$. Obviously, $C_{X}(S) \subseteq X$. Then $C_{X}(S)=X$.

ii)leftrightarrowiii) Let $S \subseteq Z(X)$ and $t \in[S, X]$. Then $t=\prod\left[s_{i}, x_{i}\right]$ such that $s_{i} \in S$ and $x_{i} \in X$. Since $S \subseteq Z(X), s_{i} \in Z(X)$ and so $t=\left[s_{i}, x_{i}\right]=0$. Therefore $[S, X]=\{0\}$. Similarity, $[X, S]=\{0\}$. Conversely, let $[S, X]=[X, S]=\{0\}$ and let $s \in S$. Therefore $[s, x]=[x, s]=0$, for all $x \in X$. Then $s \in Z(X)$. Hence $S \subseteq Z(X)$.

\section{Central IDEAL AND DeRIVED IDEAL}

In this section, we introduce the notions of central ideal and derived ideal and investigate the relation between commutative ideals and the derived subalgebra, central ideal and derived ideal and others ideals of BCI-algebras.

Definition 5. The generated ideal by $Z(X)$ is called the central ideal of $X$ and is denoted by $C I(X)$. i.e.,

$$
C I(X)=<Z(X)>=\bigcap_{Z(X) \subseteq I} I .
$$

Also the generated ideal by $X^{\prime}$ is called the derived ideal of $X$ and is denoted by $D I(X)$. i.e.,

$$
D I(X)=<X^{\prime}>=\bigcap_{X^{\prime} \subseteq I} I,
$$

where $I$ is any ideal of $X$.

Example 8. i) In Example 7 we have $C I(X)=X=D I(X)$ and for Example 5 (ii), we have $C I(X)=\{0, a, c\}, D I(X)=\{0, b\}$. 
ii) Let $X=\{0, a, b, c, d\}$ be a $B C I$-algebra in which $*$ operation is defined by the following table:

\begin{tabular}{l|lllll}
$*$ & 0 & $a$ & $b$ & $c$ & $d$ \\
\hline 0 & 0 & 0 & 0 & 0 & 0 \\
$a$ & $a$ & 0 & 0 & $a$ & 0 \\
$b$ & $b$ & $a$ & 0 & $b$ & 0 \\
$c$ & $c$ & $c$ & $c$ & 0 & $c$ \\
$d$ & $d$ & $d$ & $d$ & $d$ & 0
\end{tabular}

By routine calculations, we obtain $Z(X)=\{0, c\}$. The central ideal of $X$ is $C I(X)=$ $\{0, c\}$. Also $X^{\prime}=\{0, a, b\}$. Therefore $D I(X)=<X^{\prime}>=\{0, a, b\}$.

Theorem 15. Let I be an ideal of $X$. Then the following hold:

i) $X / I$ is commutative if and only if $X^{\prime} \subseteq I$,

ii) $X / D I(X)$ is commutative,

iii) If $D I(X)=X^{\prime}$, then $X^{\prime}$ is smallest ideal of $X$ such that corresponding quotient algebra is commutative.

Proof. i) see Theorem 5.1 in [17].

ii) Since $X^{\prime} \subseteq D I(X)$ by (i) $X / D I(X)$ is a commutative BCI-algebra.

iii) Let $D I(X)=X^{\prime}$, then $X / D I(X)=X / X^{\prime}$ is commutative. Now, let $I$ be an ideal of $X$ such that $X / I$ is commutative and $I \subseteq X^{\prime}$. Since $X / I$ is commutative by (i) $X^{\prime} \subseteq I$. Therefore, $X^{\prime}=I$. Hence $X^{\prime}$ is smallest ideal of $X$ such that corresponding quotient algebra is commutative.

Remark 5. Since $X^{\prime}$ is a subalgebra of $X, D I(X)=<X^{\prime}>$ is a closed ideal [3]. Then $X / D I(X)$ is a commutative BCI-algebra. Hence $D I(X)$ is a commutative ideal. The converse of this statement is not correct, for example $X$ is a commutative ideal of $X$ but is not a derived ideal of $X$, generally.

The following example shows that the central ideals and derived ideals are different from the other ideals, in general.

Example 9. i) Generally, a central ideal is neither commutative nor positive implicative ideal of $X$. The central ideal from Example 3 is $C I(X)=\{0, c\}$. Since $a * d=0 \in C I(X)$ but $d *(a *(a * d))=d \notin C I(X), C I(X)$ is not a commutative ideal. Also $(b * a) * a=0 \in C I(X)$ and $a * a=0 \in C I(X)$ but $b * a=a \notin C I(X)$. Therefore $C I(X)$ is not a positive implicative ideal. Also $(a *(d * a)) * c=0 \in C I(X)$ and $c \in C I(X)$ but $a \notin C I(X)$. Therefore, $C I(X)$ is not an implicative ideal. Since, $b \wedge d=d *(d * b)=d * d=0 \in C I(X)$ but $d \wedge b=b *(b * d)=a \notin C I(X), C I(X)$ is not a normal ideal. $C I(X)$ is not prime ideal of $X$, because $b \wedge d=d *(d * b)=$ $d * d=0 \in C I(X)$ but neither $b \in C I(X)$ and nor $d \in C I(X)$. 

lows

ii) Let $X=\{0, a, b, c, d\}$ be a $B C I$-algebra in which $*$ operation is defined as fol-

\begin{tabular}{c|ccccc}
$*$ & 0 & $a$ & $b$ & $c$ & $d$ \\
\hline 0 & 0 & 0 & 0 & 0 & 0 \\
$a$ & $a$ & 0 & $a$ & 0 & 0 \\
$b$ & $b$ & $b$ & 0 & 0 & 0 \\
$c$ & $c$ & $c$ & $c$ & 0 & 0 \\
$d$ & $d$ & $d$ & $d$ & $c$ & 0
\end{tabular}

By routine calculations, the central ideal of $X$ is $C I(X)=\{0\}$. Also $X^{\prime}=\{0, a, b\}$. Therefore, the derived ideal of $X$ is $D I(X)=\{0, a, b\}$. In this example $C I(X)=\{0\}$ is not a maximal ideal of $X$, because $C I(X) \subsetneq I=\{0, a\}$. Also $C I(X)=\{0, a\} \cap\{0, b\}$ but $C I(X) \neq\{0, a\}$ and $C I(X) \neq\{0, b\}$. Then $C I(X)$ is not an irreducible ideal of $X$. Since $a, b \notin C I(X)$ and neither $a * b \in C I(X)$ and nor $b * a \in C I(X), C I(X)$ is not an obstinate ideal of $X$.

iii) In Example 3, $X$ is an implicative, commutative, positive implicative, prime, obstinate, maximal, Varlet, irreducible and normal ideal but is not central ideal of $X$ (for more details see [3,12]).

Theorem 16. Let $X$ be a BCI-algebra. Then

i) $C I(X)=\left\{x \in X:\left(\ldots\left(\left(x * a_{1}\right) * a_{2}\right) * \ldots\right) * a_{n}=0\right.$, for some $\left.a_{1}, \ldots, a_{n} \in Z(X)\right\}$.

ii) $D I(X)=\left\{x \in X:\left(\ldots\left(\left(x * a_{1}\right) * a_{2}\right) * \ldots\right) * a_{n}=0\right.$, for some $\left.a_{1}, \ldots, a_{n} \in X^{\prime}\right\}$.

Proof. Since 0 is a positive element of $X$ and $0 \in Z(X)$ and $0 \in X^{\prime}$ by Theorem 3 (i) and (ii) holds.

Theorem 17. Suppose that I is a closed ideal of X. Then I is a commutative ideal if and only if $[x, y] \in I$, for all $x, y \in X$.

Proof. Let $I$ be a closed ideal of $X$. $I$ is a commutative ideal if and only if $X / I$ is a commutative BCI-algebra if and only if $X^{\prime} \subseteq I$ if and only if $[x, y] \in I$, for all $x, y \in X$.

In the following theorem we consider a condition under which the equality of Theorem 12 is correct.

Theorem 18. Let $I$ be a commutative closed ideal of $X$ and $I \cap X^{\prime}=\{0\}$. Then $I \subseteq Z(X)$ and so $Z(X / I)=Z(X) / I$.

Proof. Let $I$ be a commutative closed ideal of $X$ and let $x \in I$. Then $[x, y] \in I$, for every $y \in X$. Since $[x, y] \in X^{\prime}$ for every $x, y \in X,[x, y] \in I \cap X^{\prime}=\{0\}$. Therefore, $[x, y]=0$. Similarity, $[y, x]=0$. Thus $[x, y]=[y, x]=0$. Hence $x \in Z(X)$. That is $I \subseteq Z(X)$. But $Z(X / I)=\{x / I:[x / I, y / I]=[y / I, x / I]=0 / I$, for all $y / I \in X / I\}=$ 
$\{x / I:[x, y] / I=[y, x] / I=0 / I$, for all $y \in X\}=\{x / I:[x, y],[y, x] \in I$, for all $y \in X\}$. Since $[x, y],[y, x] \in X^{\prime}$, for all $x, y \in X$, then the recent set is equal $\{x / I:[x, y],[y, x]$ $\in I \cap X^{\prime}$, for all $\left.y \in X\right\}=\{x / I:[x, y]=[y, x]=0$, for all $y \in X\}=\{x / I: x \in Z(X)\}=$ $Z(X) / I$.

Theorem 19. If I is a commutative closed ideal of $X$, then I is a normal ideal of $X$.

Proof. Let $I$ be a commutative closed ideal of $X$ and $x \wedge y \in I$. Then $[y, x]=$ $(y \wedge x) *(x \wedge y) \in I$ for all $x, y \in X$. But $I$ is an ideal, then $(y \wedge x) \in I$.

Conversely, let $y \wedge x \in I$. Since $[x, y]=(x \wedge y) *(y \wedge x) \in I$ and $I$ is an ideal of $X$, then $x \wedge y \in I$. Therefore, $I$ is a normal ideal of $X$.

In the following example we show that the converse of Theorems 18, 19 are generally not correct.

Example 10. Let $X=\{0, a, b\}$. Define a binary operation $(*)$ on $X$ by

\begin{tabular}{l|lll}
$*$ & 0 & $a$ & $b$ \\
\hline 0 & 0 & 0 & 0 \\
$a$ & $a$ & 0 & 0 \\
$b$ & $b$ & $b$ & 0
\end{tabular}

$X$ is a BCI-algebra. The set $I=\{0\}$ is a closed ideal of $X$. With simple calculations we obtain $Z(X)=\{0\}$ and $X^{\prime}=\{0, a\}$. Also $I \cap X^{\prime}=\{0\}$ and $I \subseteq Z(X)$ but $I$ is not a commutative ideal, because $[a, b]=a \notin I$.

In the Wronski algebra [3] $I=\{0\}$ is a normal ideal of $X$ but is not a commutative ideal.

Remark 6. The set $N_{k}(X)=\left\{x \in X: 0 * x^{k}=0\right\}$, where $k$ is a fixed natural number is a commutative closed ideal of $X$ [3]. Then $X / N_{k}(X)$ is a commutative BCI-algebra. Hence by Theorem 15, $X^{\prime} \subseteq N_{k}(X)$ and so $\left.D I(X)=<X^{\prime}\right\rangle \subseteq<N_{k}(X)>=N_{k}(X)$. Therefore, any pseudo commutator element of $X$ is a nilpotent element of $X$. Also by Corollary 3 , we have $Z\left(X / N_{k}(X)\right)=X / N_{k}(X)$.

In the last theorem a relationship between $\operatorname{Rad}(X)$ and $C I(X), D I(X)$ is expressed and proved.

Theorem 20. $C I(X) \cap D I(X) \subseteq \operatorname{Rad}(X)$.

Proof. It is sufficient to show that every maximal ideal $M$ of $X$ is contains $C I(X) \cap D I(X)$. Since $M$ is a maximal ideal, $D I(X) \subseteq M$ or $X=M \times D I(X)$, because if $D I(X) \nsubseteq \subseteq M$, then $M \subsetneq M \times D I(X)$, by the maximality of $M$ in this case $X=M \times D I(X)$. If $D I(X) \subseteq M$, then $C I(X) \cap D I(X) \subseteq M$. In the case $X=M \times$ $D I(X), X / M \cong D I(X)$. Then $X / M$ is a commutative BCI-algebra and hence $X^{\prime} \subseteq M$. Therefore $D I(X)=\left\langle X^{\prime}\right\rangle \subseteq\langle M\rangle=M$. Hence $C I(X) \cap D I(X) \subseteq M$. 
Open problem: Under what conditions dose the equality in Theorem 20 hold?

\section{CONCLUSION}

In groups, rings, lie algebras, monoids and semigroups the centralizer of a subset is the set of all elements such that commute with all elements of them set and the normalizer are elements that satisfy a weaker condition. This article presented the centralizer of a subset of BCI-algebras as well as the concept of pseudo center of BCI-algebras. The results of this paper show that:

i) $X$ is commutative iff $Z(X)=X$ iff $X^{\prime}=\{0\}$.

ii) The pseudo center of $X$ is exactly $C_{X}(X)$ and $X$ is commutative if and only if $C_{X}(X)=Z(X)=X$.

iii) For subsets $S, T$ of $X, T \subseteq C_{X}(S)$ if and only if $S \subseteq C_{X}(T)$.

iv) $C_{X}\left(C_{X}(S)\right)$ contains $S$, but $C_{X}(S)$ need not contain $S$.

v) $C I(X) \cap D I(X) \subseteq \operatorname{Rad}(X)$.

Some important topics for future work are:

i) The concept of C-dimension in BCI-algebras using centralization could be an interesting subject for studies.

ii) Making normalizer of a subset $S$ of $X$ such that $N_{X}(X)=X$ and for singleton sets, $N_{X}(a)=C_{X}(a)$, for $a \in X$.

iii) Find subalgebra of $X$ to which $X / C I(X)$ (also $X / D I(X)$ ) is isomorphic.

iv) Using commutators to construct idealizer of an ideal $I$ of $X$.

\section{ACKNOWLEDGEMENT}

The authors would like to express their sincere thanks to the referee for their valuable suggestions and comments.

\section{REFERENCES}

[1] S. A. Bhatti and M. A. Chaudhry, "Ideals in BCI-algebras," Internat. J. Math. Ed. Sci. Tech., vol. 21, no. 4, pp. 637-643, 1990, doi: 10.1080/0020739900210419.

[2] W. A. Dudek, X. Zhang, and Y. Wang, "Ideals and atoms of BZ-algebras," Math. Slovaca, vol. 59, no. 4, pp. 387-404, 2009, doi: 10.2478/s12175-009-0135-2.

[3] W. A. Dudek, "On some BCI-algebras with the condition (S)," Math. Japon., vol. 31, no. 1, pp. 25-29, 1986.

[4] W. A. Dudek, “On group-like BCI-algebras,” Demonstratio Math., vol. 21, no. 2, pp. 369-376, 1988.

[5] W. A. Dudek, "Solid weak BCC-algebras," Int. J. Comput. Math., vol. 88, no. 14, pp. 2915-2925, 2011, doi: 10.1080/00207160.2011.582103.

[6] W. A. Dudek and Y. B. Jun, "Quasi p-ideals of quasi BCI-algebras," Quasigroups Related Systems, vol. 11, pp. 25-38, 2004.

[7] W. A. Dudek, B. Karamdin, and S. A. Bhatti, "Branches and ideals of weak BCC-algebras," Algebra Colloq., vol. 18, no. Special Issue 1, pp. 899-914, 2011, doi: 10.1142/S1005386711000782.

[8] W. A. Dudek and J. Thomys, "On decompositions of BCH-algebras," Math. Japon., vol. 35, no. 6, pp. 1131-1138, 1990. 
[9] W. A. Dudek and J. Thomys, "On some generalizations of BCC-algebras," Int. J. Comput. Math., vol. 89, no. 12, pp. 1596-1616, 2012, doi: 10.1080/00207160.2012.692782.

[10] K. H. Hofmann and S. A. Morris, The Lie theory of connected pro-Lie groups, ser. EMS Tracts in Mathematics. European Mathematical Society (EMS), Zürich, 2007, vol. 2, doi: 10.4171/032.

[11] Y. Huang, BCI-algebra. Elsevier, 2006.

[12] Y. Imai and K. Iséki, "On axiom systems of propositional calculi. XIV," Proc. Japan Acad., vol. 42, pp. 19-22, 1966.

[13] K. Iséki, "An algebra related with a propositional calculus," Proc. Japan Acad., vol. 42, pp. 26-29, 1966.

[14] K. Iséki, "On BCI-algebras," Math. Sem. Notes Kobe Univ., vol. 8, no. 1, pp. 125-130, 1980.

[15] A. Najafi and A. Borumand Saeid, "Solvable BCK-algebras," Cankaya University Journal of Science and Engineering, vol. 11, pp. -, 2014.

[16] A. Najafi, "Pseudo-commutators in BCK-algebras," Pure Mathematical Science, vol. 2, no. 1, pp. 29-32, 2013.

[17] A. Najafi, A. Borumand Saeid, and E. Eslami, "Commutators in BCI-algebras," Journal of Intelligent \& Fuzzy Systems, vol. 31, no. 1, pp. 357-366, 2016.

[18] A. Najafi, E. Eslami, and A. Borumand Saeid, "A new type of nilpotent BCI-algebras," An. Ştiinţ. Univ. Al. I. Cuza Iaşi. Mat. (N.S.), vol. 64, no. 2, pp. 309-326, 2018.

[19] K. C. O'Meara, J. Clark, and C. I. Vinsonhaler, Advanced topics in linear algebra: Weaving matrix problems through the Weyr form. Oxford University Press, Oxford, 2011.

[20] L. H. Shi, “An axiom system of BCI-algebras," Math. Japon., vol. 30, no. 3, pp. 351-352, 1985.

Authors' addresses

A. Najafi

Department of Mathematics, Behbahan Branch, Islamic Azad University, Behbahan, Iran

E-mail address: najafi@behiau.ac.ir

A. Borumand Saeid

Department of Pure Mathematics, Faculty of Mathematics and Computer, Shahid Bahonar University of Kerman, Pajoohesh Sq., Imam Khomeni Highway, 7616913439 Kerman, Iran

E-mail address: arshameuk.ac.ir

\section{E. Eslami}

Department of Pure Mathematics, Faculty of Mathematics and Computer, Shahid Bahonar University of Kerman, Pajoohesh Sq., Imam Khomeni Highway, 7616913439 Kerman, Iran

E-mail address: eslami@uk.ac.ir 